\title{
Gestión y gobernabilidad de las instituciones de educación superior
}

\author{
Luis Enrique Orozco*
}

\section{Introducción}

$\mathrm{H}$

aremos algunos planeamientos que inciten al diálogo posterior y permitan acrecentar nuestros conocimientos sobre el tema y aumentar la motivación hacia la problemática asociada a la gestión de la Universidad.

Para comenzar recordemos el ámbito propio de la gestión de las universidades. Al hablar de gestión nos vamos a concentrar en los fenómenos asociados a la existencia o no en una institución de Educación Superior de una estructura de poder y de reglas de juego claras para los diferentes estamentos que integran la institución. Adecuación de los talentos humanos de que dispone la institución, de su infraestructura, equipos y financiamiento para el logro de sus tareas de dirección. Estrategias de planeación y criterios de distribución presupuestal. Existencia de bases de datos y de sistemas de monitoreo del cumplimiento de las tareas sustantivas de la institución. En opinión de usuarios, grado de coherencia y calidad de la dirección de las unidades académicas y administrativas de primer nivel. Partimos de la hipótesis de que cuando en una institución todos estos elementos se encuentran consolidados, coherentes con su misión institucional y con gran aceptación por parte de los actores de la organización, tal institución posee un alto grado de gobernabilidad.

Vamos a concentrarnos en tres dimensiones del problema. De una parte el planteamiento teórico asociado a la gestión del conocimien-

\footnotetext{
* Director de la Cátedra de la UNESCO-Universidad de los Andes-Bogotá y Asesor de las Instituciones Salesianas de Educación Superior-IUS
} 
to, toda vez que una Universidad es una organización social que puede entenderse como una "empresa del conocimiento". En segundo lugar, abordaremos el tema de la gobernabilidad como expresión de una buena gestión y finalmente abordaremos el problema metodológico asociado al estudio de la gestión universitaria.

\section{La gestión del conocimiento}

En la época a de los griegos se tenía una visión unitaria del mundo y una concepción homogénea del conocimiento. La ciencia era considerada en su unidad como una especie de doble de la realidad. El conocimiento era para el hombre un fin en sí mismo, deseable en sí y por su capacidad para el desarrollo del hombre en su especificidad. El conocimiento no era un medio para alcanzar riquezas, no tenía una finalidad práctica, utilitaria. El conocimiento por excelencia era el conocimiento de las cosas en sí mismas: la metafísica.

La teoría era producto de un acto contemplativo (especulación) y estaba orientada e inspirada por la idea de "bien". El teórico era quien habiendo dedicado su vida a la especulación desinteresada, tenía el interés de servir a la polis; para que en esta última los ciudadanos pudieran reproducir el orden inmanente del cosmos, en la acción. No había una separación entre teoría y práctica, ni entre teoría y experiencia, ni entre saber y bien. Por lo tanto, el comportamiento ético era una implicación del conocimiento ${ }^{1}$.

Con la modernidad se resquebraja esta homogeneidad de la realidad y del conocimiento y se separa saber y experiencia, saber y acción y teoría y comportamiento ético. En adelante, la especulación cede su lugar a la observación. Surgen las ciencias experimentales y, con ellas un nuevo concepto de conocimiento y en especial de "conocimiento científico". Emerge la posibilidad y la exigencia de método, de ser neutrales frente a los fenómenos de la experiencia. La neutralidad del hombre de ciencia será prenda de cientifícidad. La matematización e idealmente, la axiomatización de teorías será la aspiración primera de todo saber empírico².

\footnotetext{
1 Véase: J. Habermas, Conocimiento e interés.

2 Descartes: El Discurso del Método (existen diferentes ediciones). M. Weber, El Político y el Científico. Edit. Grijalbo. México. 1989.
} 
En este contexto se desarrollan las ciencias de la naturaleza; pero también las ciencias del hombre o sociales. Bajo el paradigma de las primeras, estas últimas tendrán que definir su estatuto teórico (su carácter de ciencias). Más allá del renacimiento, época en que se vuelve a los clásicos, al saber general y básico, toda la modernidad será un esfuerzo continuado por obtener conocimientos cada vez más exactos de los fenómenos naturales, históricos y sociales (recordar, por ejemplo, en el caso de la de la Historia a Droysen, Wildelbant, Dilthey o, en la problemática asociada a la distinción entre "explicación" y "comprensión" en las ciencias) $)^{3}$.

Pero el concepto de ciencia unitario de la época moderna, tan distinto al concepto de "unidad" de los griegos se resquebraja en los finales del S. XIX y principios del XX. La crisis de las matemáticas, de la física y el surgimiento de la física cuántica, ponen sobre el tapete la discusión sobre el determinismo e indeterminismo; sobre el modelo de causalidad, como principio fundante de la ciencia ${ }^{4}$.

Paulatinamente, la discusión se desplaza a los "paradigmas" a través de una discusión sobre la historia de la ciencia. Discontinuidad versus continuidad; ruptura epistemológica, crisis, revolución, nuevos paradigmas, son todas estas categorías que desplazan y fragmentan la unidad moderna de la ciencia. Solo permanecen campos del saber, disciplinas, programas de investigación, proyectos ${ }^{5}$. Ya se perfilan estilos de universidad en correspondencia con la manera como institucionalizan y gestionan el conocimiento ${ }^{6}$. Estilos bien diferentes a los denominados modelos clásicos, desarrollados por los clásicos del pensamiento universitario ${ }^{7}$.

Al socaire de tal fragmentación emergen y se desarrollan las profesiones en un proceso que va configurando fragmentos de saber

3 Dilthey."Introducción a las Ciencias del Espiritu": y M. Weber, El Problema de la irracionalidad en las Ciencias Sociales.

${ }^{4}$ Nagel: Estructura lógica de la Ciencia. Edit. Paidos. Bs. As. 1996.

5 Th. Kuhn, Estructura de las Revoluciones Cientificas. F.C. E. México 1998.

6 M. Gibbons. Las nuevas formas de producción del conocimiento. Edit. Pomares. México 1998.

7 Fichte, Schelling y Otros. La idea de la Universidad en Alemania. Ed. Suramérica. Bs. As. 1959. 
cada vez más especializados con credenciales para el desempeño de ocupaciones y oficios en la sociedad.

En otra dimensión, las universidades que surgen hacia el S. XII -XIII emergen a la imagen de los gremios y paulatinamente se van convirtiendo en sólidas corporaciones de profesores y estudiantes interesados en la producción del conocimiento, en la formación de las personas y en el servicio a la sociedad, mediante la preparación de profesionales en los campos del derecho, la teología, la filosofía y la medicina. En su primer momento; sin embargo, su currículum estuvo centrado en el Trivium (gramática, retórica y lógica) y el quadrivium (aritmética, geometría, astronomía y música), denominadas las Artes Liberales y a los cuales se agregaron luego los estudios de la filosofía y la teología, la medicina y el Derecho. Así surge el concepto de las facultades. A su vez, debe recordarse para entonces, se trataba, hasta cierto punto, de un saber desinteresado, el "amor sciendi" e inclusive, hasta cierto punto "irresponsable". La capacitación para un oficio u ocupación era algo secundario; se trataba más bien, de la transmisión y desarrollo del saber científico y humanístico. Paulatinamente, y con el correr del tiempo fue desplazándose, según preferencias, institucionales, hacia una determinada predilección: investigación, capacitación o formación del carácter y de la personalidad del estudiante o servicio a la sociedad. Este fue el principio de los denominados estilos de universidad .

La preocupación de la Universidad por los problemas de la sociedad surge posteriormente hasta tornarse en el presente la preocupación primera. En efecto, con el surgimiento de los Estados-Naciones fue necesario para la supervivencia de estos alcanzar una identidad nacional y afirmarse como naciones en el contexto del mundo. Las universidades sirvieron a ello a su manera y, en cada país, según procesos propios.

Adicionalmente debemos tener presente la constitución de los aparatos educativos a partir de Napoleón en adelante, en virtud de los cual las universidades se convierten en centros importantes de los sistemas educativos en cada país. Con esta circunstancia, surge una doble lógica que aquellas deben atender: la lógica del establecimiento, al lado de la lógica de las disciplinas. Así, aquellas corporaciones primeras se fueron convirtiendo en entes oficiales, en sistemas educativos y al socai-

8 K. Jaspers, Véase: Fichte, Schelling y Otros. La Idea de la Universidad en
Alemania. Ed. Suramericana. 184 
re del desarrollo de cada país, fueron adquiriendo un rostro propio, convirtiéndose en elemento más o menos trascendente en el desarrollo de los países ${ }^{9}$.

Si unimos estos dos ejes de la reflexión: de una parte la evolución de la concepción y distribución de los saberes y de otra el surgimiento de los aparatos educativos, podemos decir que a finales de la modernidad, la universidad como institución refleja en su ser y en su operación el mundo externo que la circunda tanto en lo que se refiere al conocimiento como a las dinámicas de transformación de la sociedad. Ya no es el mundo idílico medieval de su origen. La institución manifiesta, el malestar de la cultura y de la sociedad; en ella se expresan las dinámicas de transformación de la sociedad ${ }^{10}$.

De esta manera, surgen para la universidad problemas nuevos derivados de la dinámica de la ciencia y del desarrollo de la sociedad: reorganización de los saberes; nuevos paradigmas; nuevas formas de producción del conocimiento; demanda sociales nuevas; desarrollo tecnológico; mayor interdependencia de los Estados; nuevas formas de aprendizaje; la educación permanente; manifestaciones en su seno de la heterogeneidad étnica, cultural y religiosa; la presencia de la mujer en la universidad; el surgimiento de nuevas profesiones; los desarrollos de la informática y de las comunicaciones. Se pasa, por lo tanto, de una institución relativamente simple en su modo de operar a una organización compleja.

Todas estas dinámicas interrogan el trabajo de las universidades de hoy. La sociedad se pregunta por la aplicabilidad del conocimiento; aparecen nuevos centros productores de ciencia y disminuye el monopolio de la Universidad en este campo; la innovación no siempre procede del sector académico; la ciencia se institucionaliza al convertirse en variable del desarrollo; los mercados laborales, cada vez más segmentados, requieren nuevos perfiles profesionales; la revolución en la informática y en las comunicaciones torna obsoletos los métodos de docen-

9 Carlos Tunnermann; Aproximación Histórica a la Universidad y su problemática actual. Universidad de los Andes. Bogotá. 1998

10 Carmen García Guadilla, Situación y Principales Dinámicas de Transformación de la Educación Superior en América Latina. Luis Enrique Orozco. (Compilador) Educación Superior. Desafío global y respuesta nacional. Ed. Omega. Bogotá. 2002. 
cia y los esquemas tradicionales de gestión; la sociedad exige que las universidades satisfagan sus necesidades de profesionalización, aumenten el nivel cultural de la sociedad y preparen una alta inteligencia para la ciencia y la tecnología. En una palabra, la pertinencia del quehacer de la universidad en lo relacionado con sus funciones sustantivas se encuentra en cuestión ${ }^{11}$.

La educación, cuya importancia se pone de presente con relación a las exigencias de un mundo globalizado, es una cuestión de "dignidad social". El aporte de la educación al desarrollo de cada país, es condición indispensable para aumentar índices de producción, para mejorar productividad, para implementar la riqueza, pero sobre todo para elevar el talento de las personas. En un mundo movilizado por el conocimiento y en el interior de instituciones cuya tarea es el conocimiento en sus múltiples formas y figuras los interrogantes del medio externo ponen en crisis su identidad. De aquí la necesidad de replantear, para el futuro, su viabilidad y gobernabilidad ${ }^{12}$.

En relación con el tema que nos concierne, debemos señalar que la actual institucionalización de la universidad no es coherente con lo que ocurre en la "sociedad del conocimiento y con sus implicaciones en términos de gestión del conocimiento en un mundo globalizado; pero tampoco en relación con los nuevos recursos de gestión moderna de las organizaciones. El cuestionamiento va más allá de justificar un estilo u otro de gestión; sino de redefinir el perfil de la institución que queremos. Hay una preocupación creciente por la cultura organizacional, y no es cosa de "reingeniería" o cosa parecida; tiene que ver con los modos de pensar, de producir conocimiento, de enseñar; es decir, con la "cultura epistemológica"13.

\footnotetext{
11 Hernando Gómez Buendía. PNUD, Educación, La Agenda del Siglo XXI.

12 Luis Enrique Orozco Silva, Educación, Modernidad y Desarrollo Humano.

13 Luis Enrique Orozco Silva, Simonía y Mercado de Ilusiones. Del mismo Autor: Formación de una Alta Inteligencia para la Ciencia y la Tecnología. El autor es conciente del carácter provisional de las consideraciones hechas en este acápite del texto.
} 


\section{La gobernabilidad de las instituciones de educación superior.}

La gobernabilidad de una institución de Educación Superior tiene que ver con la existencia o inexistencia de estructuras que permitan el logro de la misión y objetivos que se propone y con la distribución del poder y de la autoridad en ella. La gobernabilidad no es otra cosa que una forma de alcanzar resultados. Para ello el diseño institucional debe ser coherente con los fines propuestos, según el carácter académico y jurídico de la organización. En el caso de una Universidad, tales objetivos tienen que ver con sus funciones fundamentales de satisfacer la necesidad de profesionalización del país, la producción de conocimiento, la articulación con las demandas sociales y el incremento del nivel cultural de cada país.

Gobierno y gobernabilidad se complementan mutuamente. Puede existir el primero sin la segunda. La distribución del poder se hace visible en la existencia de órganos diferentes tanto personales como colegiados que posean la autoridad institucional para la toma de decisiones y que sean accesibles a los miembros que integran la institución.

Es útil, para entender analíticamente el problema de la gobernabilidad, hacer una distinción entre gobernabilidad interna y externa. Es decir entre la dimensión de la relación de las instituciones con el Estado y aquella referida a lo que ocurre en el interior de las organizaciones educativas.

En relación con la primera, ya han sido estudiados con alguna profundidad en la literatura los denominados modelos clásicos, a saber: el gobierno colegiado; el gobierno burocrático y el gobierno político. Sus características son diferentes:

En el gobierno colegiado, se trata de un esquema colectivo con base en el respeto al conocimiento en sí mismo y a la especialización. Con gran tendencia a la igualdad y el consenso. Suele propiciarse la existencia de comités que permiten y favorecen la participación y el seguimiento por parte de todos de reglas de juego que hacen viable el proyecto institucional. Aunque existen instancias de autoridad claras, se da un interés compartido por todos los estamentos en el "bien común" de la organización; y la institución como un todo tiende a ser horizontal. La manifestación de este estilo de gobierno consiste en la predilección de:

Toma de decisiones colaborativa.

Toma de decisiones consultadas. Aunque las decisiones permanezcan en quien mantiene el cargo, se consulta y respeta en buen grado la opinión de los académicos y otros organismos, según el caso. 
Existencia de grupos específicos que son responsables de temas específicos.

En el gobierno burocrático, la estructura de la organización conserva una jerarquía formal. Los cargos tienen poder y lo ejercen y canales de comunicación formales. Entre sus características principales podemos encontrar las siguientes, entre otras:

Los cargos se definen en función de competencias.

Las personas son nombradas y no elegidas.

El rango de cada persona se reconoce y respeta.

La carrera es exclusiva.

El estilo de vida de las personas está centrado en la organización.

Existe estabilidad laboral y esta está mediada por el ordenamiento profesoral o carrera académica.

El gobierno político consiste en un estilo de gobierno integrado por juntas directivas, consejos, entre otros, que involucran a los miembros de la institución tanto como a personas externas. Casi siempre se inspiran en modelos teóricos, que a su vez ofrecen la base para considerar la institución como un reflejo de la sociedad en que se encuentra; y al menos en alguna de sus vertientes, el conflicto, la existencia de grupos de intereses y la oposición entre éstos constituyen la médula organizacional y matiza, por ello mismo, el rostro propio de la institución. Pesan mucho, en su dinámica interna los conflictos sociales externos y en ocasiones reproducen la lucha de grupos de intereses exteriores a la institución. Una característica central de este modelo consiste en el predominio de los grupos de intereses y el poder corporativo en la fijación de políticas, que con frecuencia, una vez establecidas no se cumplen. La negociación cumple un papel importante en la identificación de procesos y funciona, de manera análoga a un sistema político.

Identificados estos esquemas básicos de estilo de gobierno, es importante poder identificar las variables básicas de una buena gobernabilidad. Entre estas debemos tener en cuenta las siguientes:

Corresponsabilidad en la conformación de los programas académicos y en la calidad de los mismos. Esto exige que la institución posea un PEI y un Plan de desarrollo bien preciso y compartido.

Existencia de mecanismos de rendición de cuentas de todos los estamentos de la institución. 
Disponer de una estabilidad financiera institucional para garantizar la viabilidad de la misma.

Existencia de prácticas democráticas en la toma de decisiones.

Existencia de reglas de juego compartidas y observadas (reglamentos y Estatutos).

Existencia de procesos de elección y nombramiento basados en la competencia de las personas (directivos, profesores y estudiantes).

Condiciones laborales adecuadas.

Inteligencia del entorno.

Eficiencia de las políticas y adecuada conducción de las mismas.

Existencia de múltiples alternativas para la toma de decisiones.

Existencia de condiciones para crear confianza entre los miembros y estamentos de la organización.

Pero, ¿qué tan gobernables son nuestras instituciones de Educación Superior en la Región? Existen pocos estudios al respecto; aunque la percepción dominante es que las limitaciones en materia de efectividad de la cual se acusa a la universidades, sobre todo del sector público, vincula la ineficiencia a no gobernabilidad, con una marcada incidencia sobre las condiciones extremas en materia de financiamiento Una mala gestión (asociada a no gobernabilidad), en situación de recesión generalizada, pone a las instituciones en una situación de extrema penuria. Casos hay en que el poder corporativo, al reducir su lucha a la conservación de privilegios logrados en materia pensional u otros, tienen secuestrada la Universidad.

Volvamos la mirada sobre la gobernabilidad externa; es decir, aquella que tienen que ver con la relación de las instituciones de Educación Superior con el Estado.

En esta dirección, es reconocida por todos la crisis existente. Según José Joaquín Brunner, hasta el presente el esquema que se ha seguido se mueve entre dos extremos: o bien se establece una relación sobre la base de un Estado benevolente que otorga recursos y no se preocupa por la relación costo/beneficio; o bien, se suprime la autonomía de las instituciones y se interviene de modo autoritario y sin miramiento alguno. Se señala igualmente, que entre los extremos se podría distinguir una tercera posición caracterizada por la posición del Estado como "Estado evaluador". Posiciones todas que guardan íntima relación con el contexto propio de cada país en un momento determinado. A modo de 
ejemplo, el primer esquema parece coincidir (hacia los años 60) cuando el tamaño del sistema no permitía hablar de masificación y cuando la sociedad en general reconocía el papel de la Universidad en formar una elite, otorgando por la misma razón legitimidad a su tarea. Pero hacia la década 70-80 el escenario es diferente: la matrícula en el sector se multiplica de modo inesperado en razón del surgimiento de las clases medias, del ingreso de la mujer a la educación superior con la consiguiente multiplicación de instituciones, de programas académicos, de docentes sin que hubiere habido una planeación indicativa sectorial. Hoy la situación es más aguda aún, los problemas estructurales de calidad, equidad, pertinencia, eficiencia y financiamiento se mantienen frente a un escenario cada vez más exigente, con nuevas demandas y menos legitimidad en un mundo ya globalizado y a pesar de los desarrollos logrados en materia de consolidación de grupos de investigación y de acreditación.

Así las cosas, no puede mantenerse el esquema del Estado benevolente ni optar por la alternativa de un Estado desregulador que incentive el mercado, privatizando e induciendo a las instituciones para que diversifiquen sus fuentes de ingreso; quizá se podría avanzar hacia la figura de un Estado-evaluador que buscaría orientar desde la distancia a través de la acreditación voluntaria de programas académicos; de la acreditación de instituciones con carácter obligatorio; del monitoreo de instituciones con carácter de seguimiento y apoyo para la consolidación de las instituciones y a través de la creación de un sistema Nacional de Información. Se trataría de acciones que asumen como criterio que la intervención del Estado, exigida por la carta Política, descansa sobre bases tales como: fijación de prioridades, existencia de una autonomía responsable, rendimiento de cuentas, metas pactadas con las instituciones para la fijación de recursos, creación de incentivos y valoración, por parte de las instituciones, de costos/beneficios. Así las cosas, la regulación del Estado respecto del conglomerado de las instituciones podría ser fuerte pero atento a las exigencias del ejercicio de una autonomía responsable.

Inclusive, hay quienes sostienen que el Estado podría intervenir menos sin ser por ello menos fuerte, articulando su acción sobre la base de la capacidad de autorregulación de las instituciones, favoreciendo y estimulando el rendimiento de cuentas y facilitando una mayor intervención de la sociedad civil en la fijación de las políticas. La excesiva inter- 
vención, vía la normatividad, termina ahogando la creatividad y autonomía universitaria.

Lo que queremos subrayar es el mutuo condicionamiento entre la gobernabilidad interna y externa, así como la necesidad de mirar la situación singular de cada país al respecto, dada la heterogeneidad de los sistemas de Educación Superior en la Región, y de las instituciones.

Pero, ¿qué podríamos hacer en términos generales para mejorar el grado de gobernabilidad y por ende cualificar la gestión de nuestras universidades?

Ante todo, conviene precisar que no existe un prototipo de gobierno ideal; en cada caso, dependerá de la posible combinación lograda entre un principio burocrático y un principio colegiado; en virtud de la doble lógica que compone la organización universitaria. Cada uno de ellos puede tener su riesgo propio: la politiquería, del lado de la burocracia; y la ineficiencia del lado de la colegialidad.

Hacia futuro, parece necesario hacer una renuncia colectiva en el universo de la Educación Superior de la intervención de los partidos o grupos de poder externos en la dinámica interna de las organizaciones; la politiquería le es funesta a la Universidad; asumir la eficiencia como valor en la gestión de las instituciones. Esta tarea exige afectar los intereses de las burocracias universitarias, modificar prácticas en la docencia y en las actividades de investigación, buscando ser más pertinentes frente a las diferentes demandas actuales.

También es deseable llegar a algunos consensos frente a los principios que propician un alto grado de gobernabilidad, a saber:

Propiciar la libertad académica; es decir, el derecho de cada integrante de la comunidad académica para desarrollar sus actividades propias en el campo de la docencia y la investigación. El reconocimiento de este principio no excluye el rendimiento de cuentas porque se trata de un principio que se basa en la autonomía responsable del saber, porque el servicio educativo es un bien público y porque el Estado debe velar por el bien común y la buena fe pública depositada en las instituciones que prestan este servicio.

Ejercer una gobernabilidad compartida lo que exige que se propicie la participación de los diferentes estamentos en el proceso de toma de decisiones y del conjunto de las instituciones en la conformación de las políticas públicas en materia de educación. 
Definir con claridad las reglas de juego, responsabilidades y derechos de cada quien dentro de la organización y normas precisas y minimas necesarias en la gobernabilidad externa. Respeto por el principio de la excelencia y el mérito frente a las presiones indebidas de cualquier sector que provengan.

Estabilidad financiera que evite los cambios bruscos de política y reconozca adecuadamente las exigencias de la autonomía de las universidades, consagrada en la Carta Constitucional.

Asumir la responsabilidad de rendir cuentas mediante procedimientos que permitan ver las condiciones internas de operación de las instituciones y los esfuerzos por incrementar la calidad de los servicios que ofrecen. En esta dirección, es necesario asumir el compromiso con lo público que tienen las instituciones de educación superior.

Aceptar estos principios conduce a establecer mediante consenso un esquema de gobernabilidad interna que propicie cambios en la gobernabilidad externa; es decir que favorezca la expedición de un marco legal creíble, legítimo, realista y viable, sin olvidar acciones hacia afuera que favorezcan, a su vez, la gobernabilidad interna de las instituciones.

\section{Cuestiones de método}

Con frecuencia el análisis de la gestión de las instituciones de educación superior se detiene en un esfuerzo analítico de explicación de la estructura de coordinación de aquellas por parte del Estado, con particular acento, claro está, en el problema del financiamiento. En este tema se está colonizado por lo económico. Sin embargo, es necesario prestar atención a la gestión de las instituciones hacia adentro; es decir, al conjunto, más o menos articulado, de procesos de organización y de dirección que ocurren en el ámbito de las instituciones, a la distribución $\mathrm{y}$ al ejercicio de la autoridad que se dan en ellas y el Estado ${ }^{14}$.

El campo de la gestión universitaria es amplio y complejo y, dada la naturaleza de la Universidad, su gestión trasciende el "arte de la administración empresarial" para adentrarse en las peculiaridades de la

\footnotetext{
14 José Joaquín Brunner, Evaluación y financiamiento de la Educación Superior, C.P.U., Doc. De trabajo, Santiago de Chile, 1991.
} 
producción del conocimiento, de la formación de la persona y del servicio a la sociedad. Tareas sustantivas de ayer y de hoy, que cumple el ente universitario $^{15}$. Las instituciones educativas producen un bien público, independientemente de su carácter público o privado. El bien común está en el centro de sus preocupaciones. La formación humana de las personas es su última razón de ser.

Así las cosas, surge una primera pregunta: ¿Cómo aproximarse al estudio de la gestión universitaria? La diversidad, complejidad y heterogeneidad de las modalidades de formación, de instituciones, de programas y sus diferentes historias, tamaños y tradiciones, todo lo cual se expresa en sus procesos de toma de decisiones, en la dinámica de sus conflictos, en los valores que movilizan su acción, parecería que validan el que a través de metáforas, su gestión sea inteligible. En efecto, así se ha hecho, con apoyo de las teorías organizacionales: "anarquía organizada", "caneca de basura", "cancha de fútbol de uso múltiple", entre otras ${ }^{16}$.

Cada universidad tiene su propia forma de proceder y cada nación, sus propios mecanismos de coordinación estatal; pero, a pesar de las diferencias, no habrá rasgos comunes en los mecanismos de coordinación a nivel local, regional o nacional y en los estilos de gestión institucional e interinstitucional que, una vez descritos, permitan comprender la gestión universitaria. B. Clark, ayudado del denominado "Triángulo de coordinación" busca describir tales rasgos.

En dicho triangulo, los vértices están constituidos por el Estado; el mercado y el sistema profesional (oligarquía académica). De esta manera, el conglomerado de instituciones, se ubicaría entre uno u otro vértice, haciendo menos opaca su ubicación respecto a cada uno de ellos ${ }^{17}$.

Al nivel de las instituciones, la mayoría de los estudios se centran en el análisis del poder, de los modelos de gobierno; por ejemplo, los trabajos de Cohen y March. Estos autores se aproximan a precisar una tipología de los estilos de gobierno universitario: burocrático, colegiado, polí-

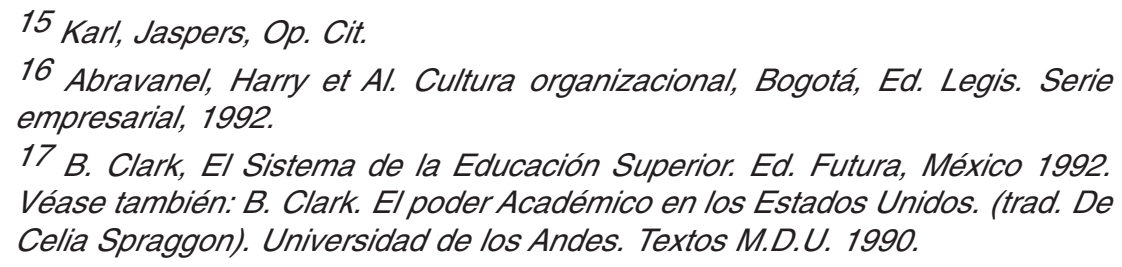
Véase también: B. Clark. El poder Académico en los Estados Unidos. (trad. De Celia Spraggon). Universidad de los Andes. Textos M.D.U. 1990. 
tico, anárquico organizado ${ }^{18}$. B. Clark integra algunos de los rasgos de estos modelos en el estudio de diferentes niveles de autoridad y en diferentes conjuntos. Por ejemplo, el departamento, la facultad, la institución, el multicampus; o también, el gobierno local, regional, o nacional ${ }^{19}$.

Pero, en su conjunto, estas aproximaciones a la gestión, como aquellas que se centran en lo financiero, son analíticas, privilegian las regularidades empíricas y, por lo tanto, lo que en la gestión es susceptible de un abordaje cuantitativo, observable, explicable. El resto, permanece en la noche oscura, en aquella dimensión sobre la cual no puede emitirse juicio serio alguno ${ }^{20}$.

Tal situación se debe, quizá, al estadio de desarrollo de los estudios en el campo de la gestión universitaria y a la crisis y estancamiento de los recursos analíticos de las denominadas Ciencias de la Administración y no solamente a razones y opciones ideológicas, o a intereses políticos subyacentes ${ }^{21}$.

Más allá de las aproximaciones analíticas es posible pensar en abordar la gestión universitaria, tomando como foco de interés los contenidos simbólicos de la gestión de las instituciones de educación superior, lo político u otro. Sobre la base de que la Universidad es una construcción social movilizada por la intención y los símbolos de quienes actúan en ella. La Universidad se crea subjetivamente y se orienta según fines y valores propios ${ }^{22}$.

En esta última perspectiva se enriquece y se hace más compleja la "comprensión" de la gestión académica; gestión que tiene que ver en alto grado con la concepción de las disciplinas, con el predominio de

\footnotetext{
18 Cohen, M:D: y March, J.G. The Process of Choise, en: M. Peterson (ed.) Organization an Governance in Higher Education. An ASHE Reader, Massachusetts, Ginn Press, 1991.

19 B. Clark Op. Cit Cap. 1.

20 Luis Enrique Orozco Silva, Teoría analítica de la ciencia y dialéctica. Universidad de los Andes. Textos MDU. Bogotá, 1992. Primera parte.

21 Omar Aktouf, La Administración entre Tradición y Renovación, Gaetan Morin Editeur, Universidad del Valle, Cali Colombia, 1996.

22 Guillermo Páramo, Consonancias y Disonancias, Luces y Sombras. La imagen del creador intelectual y la autonomía de la Universidad. Consejo Nacional de Acreditación. Bogotá, 1998.(texto en publicación).
} 
paradigmas, con la lucha ideológica y con los intereses de quienes conforman la institución ${ }^{23}$.

En general, pues, el tema de la gestión de las universidades, no vinculado a los recursos, puede dar lugar a dos enfoques: el analítico, basado casi siempre en las teorías organizacionales, o en la planeación estratégica, o en la reingeniería, o en la denominada gestión integral o en los estudios de prospectiva (planeación por escenarios) con posibilidad de tener una actitud más o menos crítica según el autor respectivo; o el enfoque histórico-crítico, inspirado en una hermenéutica que posibilita preguntarse por la racionalidad operante en el análisis, bajo los presupuestos de la "comprehensión"

Con alguna frecuencia, las posiciones del Banco Mundial ilustran el primer tipo de aproximación: Para este Organismo, la gestión es un elemento técnico de control, tanto en el nivel de la estructura de coordinación existente por parte de los gobiernos, como de las políticas puestas en marcha. De ahí, el énfasis puesto en la necesidad de redefinir las funciones de los gobiernos respecto de la educación superior con el fin de evitar una participación de estos que ha excedido con creces lo que, según el Organismo, se considera económicamente eficiente.

Las premisas para apreciar el tipo de intervención adecuada de los gobiernos serían: establecer un marco de políticas coherentes (en lo jurídico, en planeación a largo plazo, creación de organismos independientes de la fiscalización); apoyar con incentivos e instrumentos al mercado par aplicar las políticas (ejemplo: apoyo a la demanda); mayor autonomía administrativa y responsabilidad de las instituciones.

Un ejemplo más preciso, puede apreciarse en $\mathrm{R}$. Winkler, quien se interesa particularmente por la eficiencia interna y externa. Para el logro de la primera, según el autor, es necesario disponer de sistemas de información, introducir criterios de desempeño entre las unidades de cada institución, establecer sistemas de acreditación y capacitar los administradores universitarios. Para el logro de una eficiencia externa, sugiere la creación de sistemas de evaluación por pares, definición de estándares

23 Véase: Luis Enrique Orozco Silva. Op. Cit. Segunda Parte.

24 El enfoque dominante se percibe en los temas que se privilegian al estudiar la gestión universitaria y el modo propio utilizado desde las diferentes disciplinas utilizadas. 
para la aplicación de recursos y brindar una asistencia técnica regional para mejorar la calidad y la eficiencia administrativa ${ }^{25}$.

En la segunda perspectiva de análisis surgen preocupaciones de otro orden. Se enfatiza, por ejemplo: la necesidad de construir consensos, la necesidad de delinear el futuro, la urgencia de disponer de valores compartidos. Ante las nuevas dinámicas de transformación en la región se señala: la necesidad de pensar el tema de la viabilidad y gobernabilidad de las instituciones; de asumir los valores de la eficiencia y de la eficacia, tanto como el de la pertinencia; el ejercicio de la autonomía responsable; la búsqueda estructuras internas horizontales, flexibles, susceptibles de responder a escenarios cambiantes; todo esto, sin perder de vista: la autonomía y la libertad académicas; la universalidad del conocimiento y las urgencias nacionales; tanto como la autor-regulación y el rendimiento de cuentas. Todo ello, orientado a una búsqueda de una reorientación de la gestión universitaria que permita: obtener resultados pertinentes y útiles a la sociedad y con visión de largo plazo; considerar el contexto para definir estrategias de integración de la institución y de su medio externo; estilo participativo, con estructuras horizontales y unidades descentralizadas de autonomía relativa; empleo asociado a opciones de auto-realización y con estímulos por resultados; descentralización financiera; control selectivo y asignación de recursos a la innovación que permita un mayor valor agregado a los procesos institucionales; modernizar y hacer gestión a través de valores o lo que es lo mismo hacer una "Dirección por Valores".

No se deduce de lo señalado anteriormente que la posición correcta sea asumir una posición metodológica frente a otra, sino más bien la necesidad de asumir con claridad el enfoque que se desee con una visión crítica de sus alcances y limitaciones. En este terreno falta mucho camino por andar y lo dicho debe tomarse como una primera aproximación al tema, todavía muy provisional. Una conclusión adecuada iría en la dirección de sostener la no neutralidad de los enfoques en materia de estudio de la gestión universitaria. Aunque no hablemos con verdades absolutas respecto del método, poner las opiniones en la palestra pública es una manera de enriquecerlas y acercarnos hacia una verdad siempre en construcción.

25 Ronald Winkler, Eficiencia financiera en la Educación Superior, en Finanzas de la Educación Superior. Universidad de los Andes. Bogotá, 1991. 